\title{
Presence of pathogenic Escherichia coli is correlated with bacterial community diversity and composition on pre-harvest cattle hides
}

Jessica Chopyk', Ryan M. Moore ${ }^{1}$, Zachary DiSpirito ${ }^{1}$, Zachary R. Stromberg ${ }^{1}$, Gentry L. Lewis ${ }^{1}$, David G. Renter ${ }^{2}$, Natalia Cernicchiaro ${ }^{2}$, Rodney A. Moxley ${ }^{3}$ and K. Eric Wommack ${ }^{1 *}$

\begin{abstract}
Background: Since 1982, specific serotypes of Shiga toxin-producing Escherichia coli (STEC) have been recognized as significant foodborne pathogens acquired from contaminated beef and, more recently, other food products. Cattle are the major reservoir hosts of these organisms, and while there have been advancements in food safety practices and industry standards, STEC still remains prevalent within beef cattle operations with cattle hides implicated as major sources of carcass contamination. To investigate whether the composition of hide-specific microbial communities are associated with STEC prevalence, 165 ribosomal RNA (rRNA) bacterial community profiles were obtained from hide and fecal samples collected from a large commercial feedlot over a 3-month period. These community data were examined amidst an extensive collection of prevalence data on a subgroup of STEC that cause illness in humans, referred to as enterohemorrhagic E. coli (EHEC). Fecal 165 rRNA gene OTUs (operational taxonomic units) were subtracted from the OTUs found within each hide $16 \mathrm{~S}$ rRNA amplicon library to identify hide-specific bacterial populations.
\end{abstract}

Results: Comparative analysis of alpha diversity revealed a significant correlation between low bacterial diversity and samples positive for the presence of E. coli O157:H7 and/or the non-O157 groups: O26, O111, O103, O121, O45, and O145. This trend occurred regardless of diversity metric or fecal OTU presence. The number of EHEC serogroups present in the samples had a compounding effect on the inverse relationship between pathogen presence and bacterial diversity. Beta diversity data showed differences in bacterial community composition between samples containing 0157 and non-0157 populations, with certain OTUs demonstrating significant changes in relative abundance.

Conclusions: The cumulative prevalence of the targeted EHEC serogroups was correlated with low bacterial community diversity on pre-harvest cattle hides. Understanding the relationship between indigenous hide bacterial communities and populations may provide strategies to limit EHEC in cattle and provide biomarkers for EHEC risk assessment.

Keywords: STEC, EHEC, O157, Non-O157, 16S rRNA gene, Microbiome, Community profiles, Cattle

\footnotetext{
*Correspondence: wommack@dbi.udel.edu

${ }^{1}$ Delaware Biotechnology Institute, University of Delaware, Delaware

Biotechnology Inst., 15 Innovation Way, Newark, DE 19711, USA

Full list of author information is available at the end of the article
} 


\section{Background}

Shiga toxin-producing Escherichia coli (STEC) strains are zoonotic pathogens that colonize the lower gastrointestinal tracts of cattle and other ruminants. STEC strains are shed in the feces of these animals, which serve as reservoirs and major sources of foodborne illness [1, 2]. In the USA, foodborne STEC infections are estimated to cause 175,905 illnesses and 21 deaths each year, with $55.2 \%$ of the outbreaks attributable to beef [3, 4]. The STEC serotype O157:H7 (E.coli O157) is highly virulent with a $46.2 \%$ hospitalization rate, compared to $12.8 \%$ for that of non-O157 STEC [3]. E. coli O157 is the most widely characterized and studied serogroup of STEC [5-7], although 20 to $50 \%$ of infections worldwide are caused by non-O157 serogroups, largely O26, O111, O103, O121, O45, and O145 [8, 9].

Every environment from the cattle gut to the human intestine is an opportunity for these pathogens to encounter and compete with other microbial populations. Commensal indigenous microbes have been shown to mitigate the proliferation of invading pathogens through predation, nutrient competition, and the excretion of antimicrobial compounds [10-12]. In particular, E. coli O157 appears to thrive within microbial communities demonstrating lower species diversity [13]. Specifically, in soil and manure environments, microbial diversity is negatively correlated with the invasion of E. coli O157 and Listeria monocytogenes [12, 14, 15]. These studies suggest that indigenous microbial populations interact, often negatively, with pathogen populations. This apparent protection against invasion is especially evident when the diversity of the microbial community is large enough to occupy a broad spectrum of ecological niches, thereby reducing the likelihood that an alien species could gain traction within the novel environment [11].

In cattle, hides are major contributors to STEC contamination of carcasses, particularly during slaughter [16-19]. As a result, STEC-positive hide samples have been shown to be more predictive of carcass contamination than fecal-positive samples [20, 21]. Interventions focused on the hide, such as dehairing, and washes with water, various chemicals, organic acids, and bacteriophage have been developed to stem STEC contamination [22]. However, the role of the commensal hide bacterial community in preventing or limiting the prevalence of STEC in cattle is unknown.

Enterohemorrhagic E. coli or EHEC, a subgroup of STEC, are generally defined as $E$. coli that contain genes that encode Shiga toxin $(s t x)$ and the locus of enterocyte effacement proteins (e.g., intimin, eae) [23]. EHEC within the $\mathrm{O} 157$ and six aforementioned non-O157 serogroups cause $>90 \%$ of the human STEC infection cases in the USA [24] and have been declared adulterants in raw, nonintact beef by the US Department of Agriculture Food
Safety and Inspection Service [25]. To examine whether a connection between EHEC presence and the composition of hide-specific bacterial communities exists, we performed high throughput $16 \mathrm{~S}$ ribosomal RNA (rRNA) sequencing of bacterial communities from hide samples exhibiting varying degrees of E. coli O157 and non-O157 contamination over a 3-month time period.

\section{Methods \\ Sample collection}

A total of 576 cattle were sampled over the course of 12 weeks in summer 2013 from a large commercial feedlot operation. Fecal samples were collected at the feedlot, while hide-on carcass surface sponge samples were collected at the abattoir as previously described [26]. Each week, 24 fresh pen-floor fecal samples were collected from each of two cattle pens 12 to $24 \mathrm{~h}$ prior to transport to the harvesting plant. At the plant, 24 hideon carcass samples were collected from cattle in each of the two study pens each week using $11.5 \times 23.0-\mathrm{cm}$ sponges (Speci-Sponge; Nasco, Fort Atkinson, WI) premoistened with $35 \mathrm{~mL}$ of $0.1 \%$ sterile buffered peptone water $(\mathrm{BPW})$. Sponges were used to sample an area of the hide of $1000 \mathrm{~cm}^{2}, 15 \mathrm{~cm}$ from the midline at the level of the diaphragm, after cattle were stunned and bled, prior to hide removal. Sampling procedures as well as characteristics of the study population were described in [26]. Aliquots of $5 \mathrm{~g}$ and $5 \mathrm{~mL}$ were removed from each fecal and hide sample, respectively, for microbiome analysis. These aliquots were snap-frozen in liquid nitrogen (LN2) within 1 and $2 \mathrm{~h}$ of collection of fecal and hide samples, respectively, and stored at $-80{ }^{\circ} \mathrm{C}$ until completion of a molecular detection assay for EHEC.

\section{EHEC detection in hide samples}

Ninety milliliter of E. coli broth (EC; Oxoid Lt, Hampshire, UK) was added to a $35 \mathrm{~mL}$ sample-BPW suspension and incubated at $40{ }^{\circ} \mathrm{C}$ for $6 \mathrm{~h}$. A previous study [27] tested the prevalence of EHEC in these hide samples using the NeoSEEK ${ }^{\mathrm{TM}}$ STEC Detection and Identification test (NS; Neogen Corp., Lansing, MI). The NS utilizes PCR and mass spectrometry to test for $>70$ independent markers including O-group, Shiga toxin, and intimin. This test determines the presence or absence of EHEC O26, O45, O103, O111, O121, O145, and O157. This work was conducted at GeneSEEK $^{\oplus}$ Inc. (Lincoln, NE). A hide sample positive for EHEC O26, O45, O103, O111, O121, or O145 was grouped into a non-O157 EHEC category, and a sample positive for both non-O157 EHEC and EHEC O157 was grouped into a category called both. A subset (180 hide samples over 8 weeks) of the 576 hide samples that tested positive by NS for EHEC O157, non-O157 EHEC, both, or negative were selected for $16 \mathrm{~S}$ rRNA bacterial community profiling. 


\section{Microbial community processing}

Microbial nucleic acids were extracted from a total of 192 fecal and 180 hide samples collected across the 12-week study via the MO BIO PowerViral DNA/RNA Isolation $\mathrm{Kit}^{\mathrm{Tm}}$. After measuring DNA concentration with the Qubit ${ }^{\circ}$ Fluorometer, the V3-V4 region of the 16S rRNA gene was amplified using dual-indexed primers [28], with an annealing temperature of $52{ }^{\circ} \mathrm{C}$ for 32 cycles. Aliquots of amplicon reactions $(3 \mu \mathrm{L})$ were electrophoresed on agarose gels to verify amplification. Amplicons were normalized with SequalPrep ${ }^{\text {Tx }}$ Normalization Plates (Invitrogen Inc., CA, USA), pooled, and then sequenced on the Illumina MiSeq platform (Illumina, San Diego, CA) using the paired-end 250 base pair sequencing protocol. Sequence data discussed in this publication have been uploaded to the DNA Databank of Japan under DDBJ BioProject accession number PRJDB4262.

\section{Microbial community analysis OTU picking and classification}

Raw Illumina sequences from hide and fecal samples were together subjected to quality control and demultiplexing using methods previously described [28]. Processed sequences were classified using open-reference OTU (operational taxonomic units-97 \% identity) picking in Qiime [29]. Briefly, the processing steps were as follows: (1) sequences were clustered against GreenGenes v13.8 [30]; (2) sequences that failed to cluster with the reference database were subsequently clustered $d e$ novo, and the centroids of these clusters were used as reference sequences for the reference database in step 3; (3) sequences that failed the first clustering were clustered against the new reference database generated in step 2; and (4) remaining unclassified sequences were clustered de novo. Clustering was performed using UCLUST [31], and resulting sequences were aligned to GreenGenes with PyNAST [29]. OTU classification was reported to the lowest possible taxonomic level.

\section{Hide-specific dataset}

A dataset containing OTUs specific to hide samples (hereby referred to as Hide Specific OTUs-HSO) was created by first identifying all OTUs that were present in any fecal sample (all fecal OTUs-AFO). These OTUs were then removed from the set of OTUs present in any hide sample, resulting in a set of OTUs that were present in hide samples, but absent from fecal samples (HSO). This hide-specific data set was analyzed in conjunction with the dataset containing all hide OTUs (all hide OTUs-AHO) (Additional file 1: Figure S1).

\section{Alpha diversity}

Community richness, to assess alpha diversity, was evaluated for each sample using the Chao1 estimator [32] and Faith's phylogenetic diversity (PD) [33]. Additionally, samples were pooled by various metadata categories (EHEC testing status of hide samples, number of EHEC serogroups, etc.) to examine differences in richness associated with each group. To assess the statistical significance of observed differences in alpha diversity for each metadata group, a jackknifying approach was used. Ten jackknifes were performed for each category, and alpha diversity metrics were calculated for each resulting subset. The level for the jackknifying was set at $95 \%$ of the number of reads of the metadata category with the fewest reads. Significant differences between jackknifed alpha diversity statistics were determined with paired Mann-Whitney tests at an alpha level of 0.05 with Bonferonni correction. These analyses were performed on both the AHO and HSO datasets.

\section{Beta diversity}

Principal coordinates analysis (PCoA) plots were generated with Emperor [34] using weighted and unweighted UniFrac [35] distance to evaluate large-scale structural shifts associated with each metadata category. Taxonomic profiles for each metadata group were analyzed with Kruskal-Wallis one-way analysis of variance and paired Mann-Whitney tests to identify taxa at significantly higher or lower abundance. OTUs demonstrating significant change were visualized with Cytoscape [36]. Beta diversity analyses were repeated for both $\mathrm{AHO}$ and $\mathrm{HSO}$ datasets.

\section{Results \\ EHEC identification}

For serogroup identification, the NS test indicated that of the 180 hide samples, 41 tested negative for any EHEC, 14 tested positive for E. coli $\mathrm{O} 157$ only, 84 tested positive for at least one non-O157 serogroup only, and 41 tested positive for E. coli $\mathrm{O} 157$ and at least one other non-O157 serogroup [27] (Additional file 1: Table S1). Fecal and hide-on carcass samples prevalence at the sample and pen-level and characteristics of the study population were presented elsewhere [26, 27].

\section{S rRNA sequencing}

After removing poor quality reads and singleton OTUs, 9,599,163 high-quality $16 \mathrm{~S}$ rRNA gene sequences were recovered from fecal $(7,910,209)$ and hide $(1,688,954)$ bacterial samples. From these sequences, 97,050 OTUs were classified across the 356 fecal and hide samples at $97 \%$ identity (nine hide samples were removed due to low quality sequencing). Hide samples provided an average of 9877 sequences per sample (Additional file 1: Figure S3), while the mean sequences per sample for fecal samples was 26,963 . In ranking the abundance of the OTUs across all hide samples, $80 \%$ of reads were contained in the top 
225 clusters, $90 \%$ in the top 554, $95 \%$ in the top 1165 , and $99 \%$ in the top 4062 (Additional file 1: Figure S4).

Feces are often found on the hide and likely influenced the occurrence of certain bacterial populations in hide samples. To ensure that trends identified in the dataset containing all hide OTUs (AHO) were robust to these effects, OTUs identified within fecal samples were subtracted from the collection of OTUs found within each hide 16S rRNA amplicon library and designated as hidespecific OTUs (HSO), and all analyses performed on the AHO dataset were repeated on the HSO dataset. After the removal of OTUs also present in fecal samples (all fecal samples-AFO), 2709 hide-specific OTUs (97 \% identity) containing 135,202 hide-specific sequences remained (mean 790.655 sequences per sample). Within this collection of sequences, a total of 2709 hide-specific OTUs (97\% identity) were identified across the hide sponge samples. Analysis for the AFO dataset is not within the scope of this manuscript and will be presented elsewhere.

\section{Bacterial community taxonomy}

The principal bacterial families identified in the hide samples (AHO) were Corynebacteriaceae, Ruminococcaceae, Lachnospiraceae, and Clostridiaceae, and of these, the Corynebacteriaceae was the largest family regardless of testing status (Additional file 1: Figure S5). When the fecal OTUs were subtracted, the dominant bacterial families in the hide-specific dataset (HSO) were Moraxellaceae, Staphylococcaceae, Streptococcaceae, and Pasteurellaceae, and of these, the Staphylococcaceae was the dominant family in the EHEC positive samples, O157 (39\%), nonO157 (32\%), and both (34\%), while the Moraxellaceae was the largest in the EHEC negative sample (33 \%) (Additional file 1: Figure S6). Streptococcaceae populations comprised a lower relative abundance in E. coli O157 (12\%) than in the non-O157 (20 \%) and EHEC negative samples (21\%). A comparison of the taxonomic distribution of bacterial species in fecal-specific (FSO), hidespecific (HSO), and shared OTUs is shown in Additional file 1: Figure $\mathrm{S} 2$.

\section{Alpha diversity metrics by testing status}

In the AHO dataset, alpha diversity was significantly lower (Mann-Whitney test, $P<0.05$ ) in cattle hide samples grouped by testing status when any of the EHEC were detected, with the lowest diversity observed in samples that tested positive for both $\mathrm{O} 157$ and nonO157 (Fig. 1). The trend continued regardless of whether the diversity was measured by Faith's phylogenetic diversity, the Chao1 species estimator, or observed OTUs (Fig. 1). Additionally, samples were pooled based on number of EHEC serogroups detected, regardless of whether they were $\mathrm{O} 157$ or non-O157 positive. When all the OTUs were present, alpha diversity was lower in samples containing one, two, three, or more serogroups as compared to EHEC negative samples (Fig. 1).

The trends seen in the AHO dataset were also seen in the HSO dataset. Alpha diversity was lower for EHEC positive samples (i.e., samples testing positive to either O157, non-O157, or both) when compared to samples without detectable EHEC (Additional file 1: Figure S7), as well as alpha diversity being lower in samples with multiple serogroups as compared to samples without EHEC (Additional file 1: Figure S7).

\section{Beta diversity metrics by testing status}

Beta diversity was compared between testing status (i.e., samples containing either O157, non-O157, or both; Fig. 2a) and the number of serogroups identified within samples (i.e., one, two, three, or more serogroups; Fig. 2b) using a three-dimensional principal coordinate analysis on the AHO and HSO datasets. Regarding the AHO dataset, along the first principal component (PC1), which explained $35 \%$ of the total variability between communities, EHEC positive samples grouped away from EHEC negative samples. Along PC2, which accounted for $36 \%$ of the total variability, samples positive for $\mathrm{O} 157$ clustered away from the other categories. When grouped by number of EHEC serogroups identified (Fig. 2b), the negative samples (0 EHEC) and positive samples (one or more EHEC) clustered away from each other along PC1 (38\% of total variability). Samples having one EHEC serogroup population had the same partitioning as $\mathrm{O} 157$ along PC1 (31 \% of total variability) as samples in the plot grouped by testing status (Fig. 2a). Within the one serogroup group, there were 53 samples $(79 \%)$ that contained a non-O157 serogroup compared to 14 samples (21 \%) with O157. Thus, the O157 group in Fig. 2a is similar to the one EHEC group in Fig. 2b. Because both groups show similar partitioning, it appeared that the presence of the O157 serotype was driving the separation.

When considering the HSO dataset (Fig. 3), there was a lack of distinct separation between the groups (data not shown). As a consequence, the weighted Unifrac measure was employed in the hide-specific analysis (Fig. 3). Along PC1, which explained $55 \%$ of the total variability, EHEC testing status grouped away from each other (Fig. 3a). Similar to Fig. 2b, the samples were grouped by the number of EHEC serogroups detected (Fig. 3b). Along PC1, which explained $73 \%$ of the total variability, the samples with one serogroup and the samples negative for all EHEC (0) clustered away from samples with two or more serogroups.

\section{OTUs demonstrating significant differences with EHEC testing status}

In our study, we use the typical convention of defining OTUs as clusters of reads at $97 \%$ similarity. Each OTU 

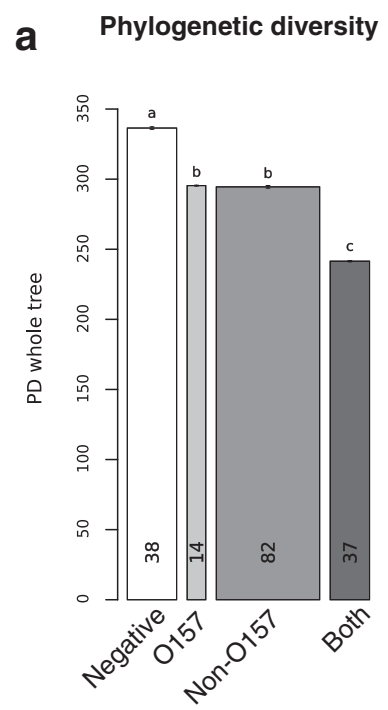

b

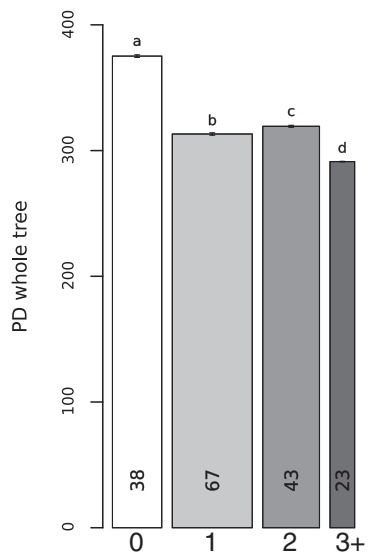

Chao1 index
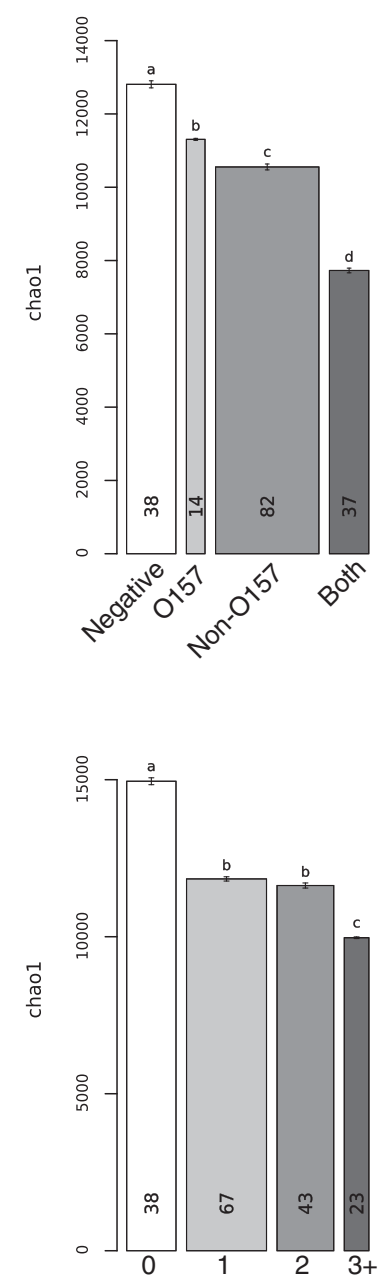

Observed OTUS
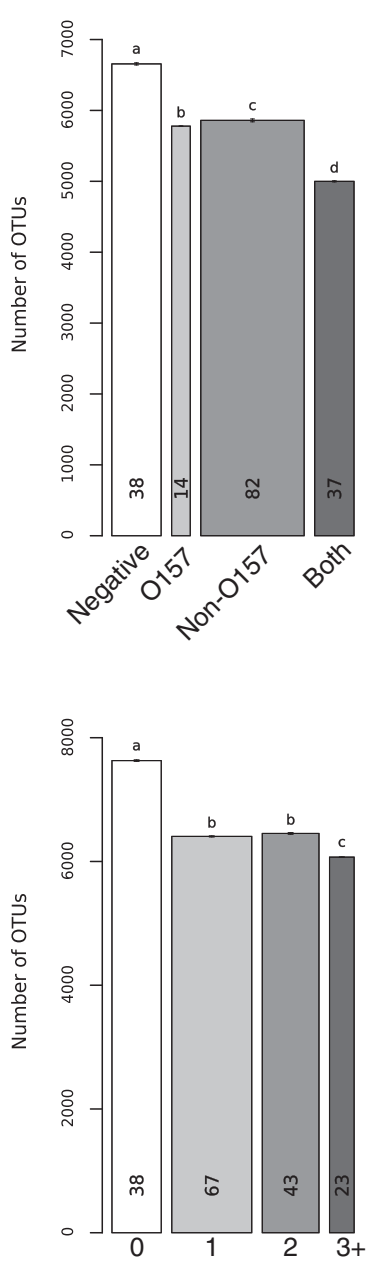

Fig. 1 Alpha diversity metrics for samples pooled by EHEC testing status for the all hide OTUs (AHO) dataset. Jackknifed alpha diversity metrics (Faith's phylogenetic diversity, Chao1 estimator, and observed OTUs) were calculated with Qiime's "alpha_rarefaction.py" script. Faith's phylogenetic diversity, the chao 1 index, and observed OTUs were calculated for ten jackknifes at a depth of $95 \%$ of the reads contained in the smallest metadata group. Error bars represent the standard error of the jackknife estimate. The width of the bar shows the number of sequences contained in each metadata category, while the small numbers inside the bars indicate the number of samples in each category. Within each chart, bars with different letters were significantly different at an alpha level of 0.05 with the Bonferroni correction as determined by the Mann-Whitney test. a Samples pooled by EHEC testing status into four groups: EHEC negative, E. coli O157 positive, non-O157 positive and positive for both E. coli O157, and any of the tested non-O157 serogroups. b Samples pooled by number or EHEC serogroups present in each sample

that follows is labeled with the most granular taxonomic classification available. OTUs occurring at significantly higher or lower relative abundance $(P<0.05)$ depending on testing status were identified in both the AHO and HSO datasets (Fig. 4). No OTUs were identified as occurring at a higher relative abundance in EHEC-positive samples in either dataset (Fig. 4), due to the decrease in diversity as depicted in Fig. 1. In the AHO dataset, the "both" group of samples had 23 OTUs at a relative lower abundance. Of these, four were shared with "O157" positive group, Streptococcus OTU \#532232, Selenomonas OTU \#311471, Mogibacteriaceae OTU \#4295063, and Rumiococcaceae OTU \#515308, and six were shared with "non-O157" positive group, Dietzia OTU \#1126467, Ruminococcus OTU \#333948 and \#532187, Thermoactinomycetaceae OTU \#4400372, Clostridales OTU \#110678 and Brevibacterium OTU \#73538. The remaining 13 were not shared with any other groups of samples that were categorized by EHEC prevalence: Lachnospiraceae OTU \#299184 and \# 294440, Jeotgalicoccus OTU \#4404401, Fusobacterium OTU \#298 7631, Trichococcus OTU \#4403115, Clostridiales OTU \#44 82650, Corynebacteria OTU \#412872, \#235898, and \#103 606, Yaniella OTU \#115315, Clostridiaceae OTU \#4383953, Butyrivibrio OTU \#13983, and SMB53 OTU \#180516. All the OTUs at lower or higher relative abundance in the EHEC negative ("none") and "non-O157" groups were 


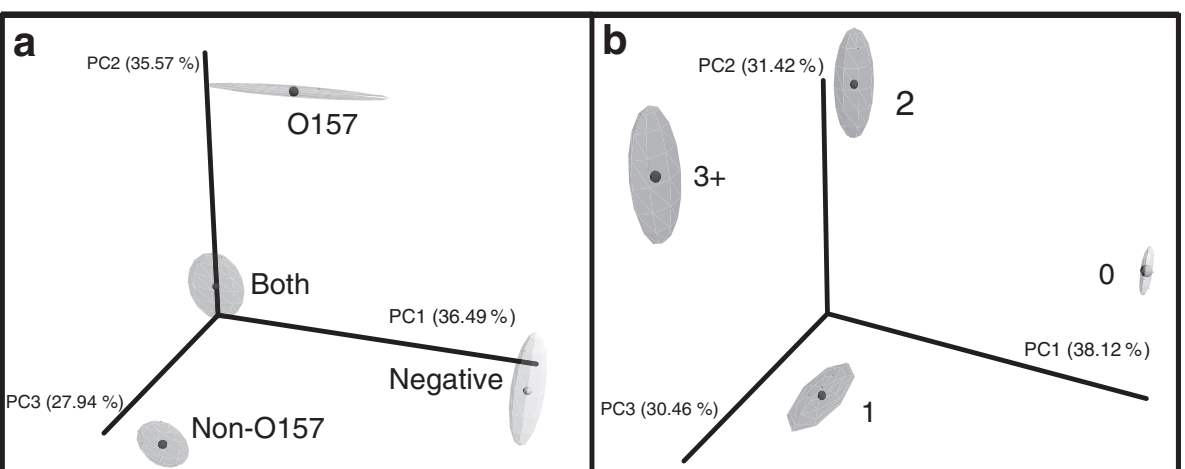

Fig. 2 Beta diversity comparison of the bacterial communities by EHEC testing status and number of EHEC serogroups for the all hide OTUs $(\mathrm{AHO})$ dataset. Jackknifed principal coordinate analysis using the unweighted UniFrac metric was performed on all hide sequences with 100 jackknifes of $95 \%$ of the reads of the smallest sample within that metadata category. a Samples pooled by EHEC testing status into four groups: EHEC negative, E. coli O157 positive, non-O157 positive and positive for both E. coli O157, and any of the tested non-O157 serogroups. b Samples pooled by number or EHEC serogroups present in each sample. Percentage of variation explained by principal coordinate shown on each of the axis

shared with another group. However, there was one OTU at lower abundance in the "O157" positive group that was not shared with any of the other EHEC metadata categories, Clostridales OTU \#560906. The EHEC negative group, "none," had three OTUs that were at greater relative abundance, which were all at lower relative abundance in "non-
O157" positive samples, Brevibacterium OTU \#73538, Ruminococcus OTU \#532187, and Nocardioidaceae OTU \#528213.

The hide-specific dataset (HSO) had fewer OTUs showing significant $(P<0.05)$ differences in relative abundance with EHEC testing status. This was due to

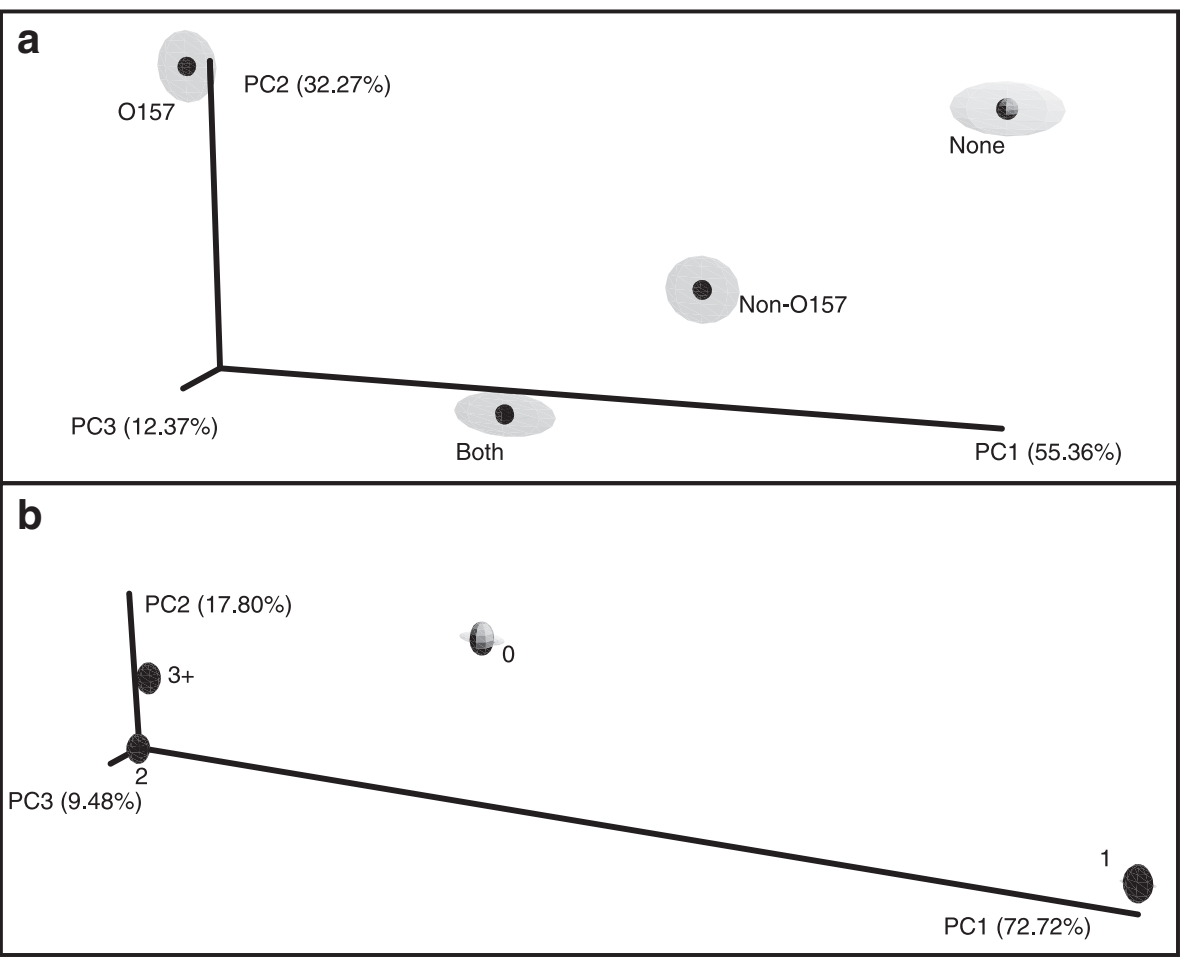

Fig. 3 Beta diversity comparison of the bacterial communities by EHEC testing status and number of EHEC serogroups for the hide-specific OTU (HSO) dataset. Jackknifed principal coordinate analysis using the weighted UniFrac metric was performed on all hide sequences with 100 jackknifes of $95 \%$ of the reads of the smallest sample within that metadata category on hide-specific OTUs. a EHEC testing status. b Number of EHEC serogroups detected in each sample. Percentage of variation explained by principal coordinate shown on each of the axis 
a

Corynebacterium

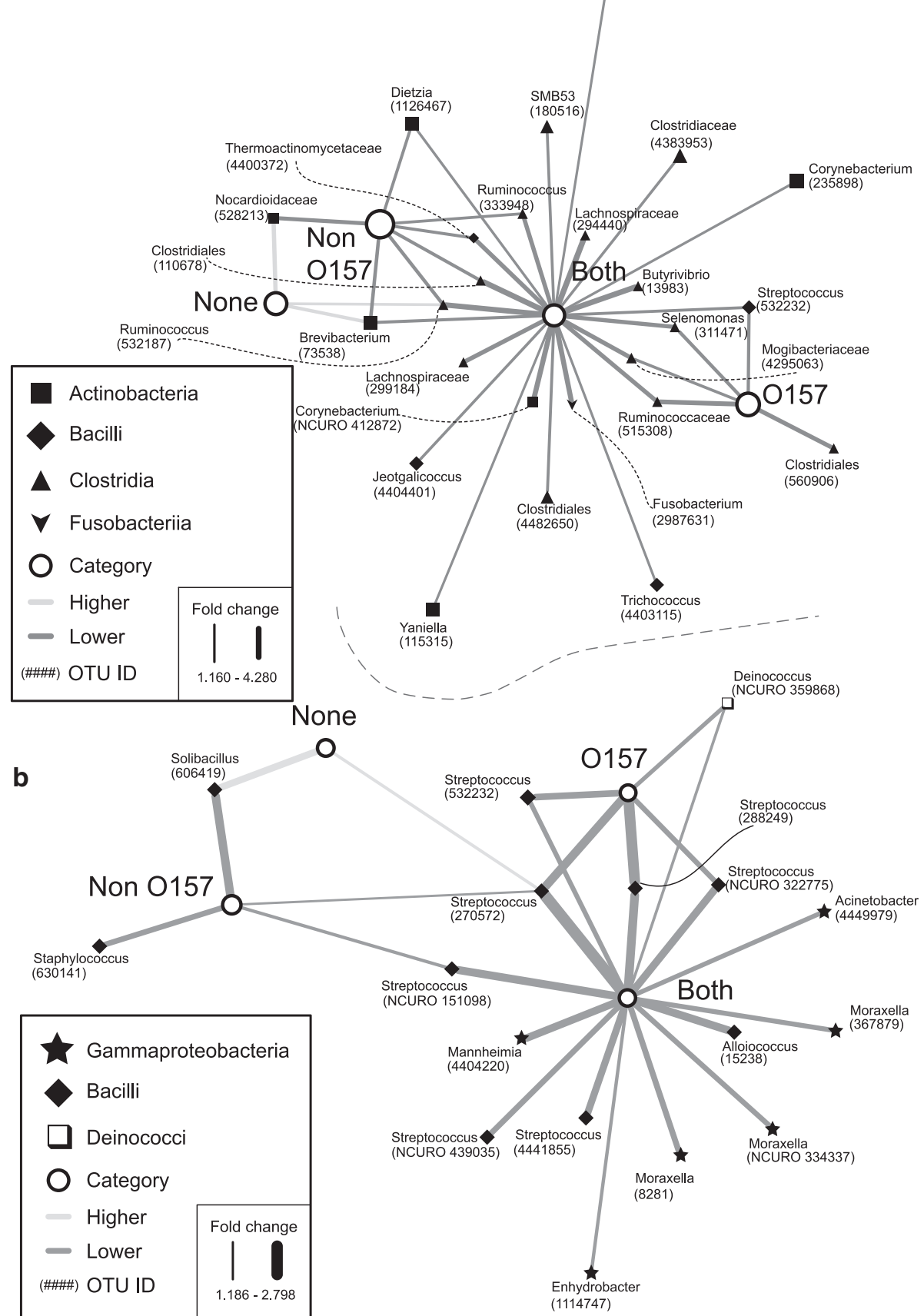

Fig. 4 OTUs with significantly different relative abundance according to tesing status. a All hide OTUs (AHO). b Hide-specific OTU (HSO). White circles indicate the testing status of groups of samples: EHEC negative; O157 only; non-O157 only; and both non-O157 and O157. Black nodes are OTUs with significantly higher $(P<0.05)$ or lower relative abundance. The shape of OTU nodes is based on class: black diamond Bacilli, black up-pointing triangle Clostridia, black square Actinobacteria, black star Gammaproteobacteria, white square Deinococci, black down-pointing triangle Fusobacteria. Labels indicate the genus of the OTU. OTU numbers from the cluster analysis are indicated in parentheses. A dark gray line represents lower average relative abundance. Light gray lines indicate higher relative abundance. Edge width is proportional to fold-change, with a thicker line representing a higher shift in abundance. When genus information was not available, family or order is indicated 
the reduction in the number of samples and OTUs in the HSO dataset (Fig. 4b). The groups were similarly distributed as in Fig. 4a, with the EHEC-positive categories ("both," "O157," and "non-O157") having only OTUs at lower relative abundance and the EHEC negative group "none" having only OTUs at higher relative abundance. The "both" group, as in the dataset containing fecal samples, had the largest abundance of altered OTUs at 15 . Five of these were shared with the "O157" and two with "non-O157" groups. Almost all of these OTUs were part of the genus Streptococcus (“O157" OTUs \#270572, \#288249, \#322775, and \#532232; "non-O157" OTUs \#270572 and \#151098), with the exception of Deinococcus OTU \#359868 shared with "O157." One of the Streptococcous OTUs (\#270572) was also at a higher relative abundance in the "none" group. The remaining nine OTUs that were at lower relative abundance in the "both" group were as follows: Mannheimia OTU \#440422, Enhydrobacter OTU \#1114747, Moraxella OTU \#8281, \#334337, \#367879, Alloiococcus OTU \#15238, Acinetobacter OTU \#4449979, and two more Streptococcous OTUs (\#4441855 and \#439035). There was also one "nonO157" OTU that was at lower relative abundance, Staphylococcus OTU \#630141, and not shared with any other group. Only one other OTU demonstrated higher relative abundance in the hide-specific "none" group, Solibacillus OTU \#606419, and was also at relative lower abundance in the "non-O157" group.

\section{Discussion and conclusions}

Current research suggests that the indigenous microbial community of the skin is on the forefront of defense against the growth of invading bacteria [37, 38]. For instance, Staphylococcus epidermidis, a commensal bacterium found on the skin of humans, has the ability to prevent the colonization of pathogens, such as $S$. aureus and group A Streptococcus, through the binding of keratinocyte receptors and the production of phenol-soluble modulins and other antimicrobial peptides [39-42]. Additionally, increased diversity on healthy intact skin in humans has been speculated to reduce the spread of opportunistic pathogens present in wounds [43]. This complex and varied ecosystem is poorly understood in cattle and may provide a rich source of information for the mitigation of pathogen spread and colonization.

Our analyses showcased the relationship between the composition of hide bacterial communities and the prevalence of EHEC contamination on cattle hides. To test whether these correlations seen in the ASO dataset were truly associated with hide bacterial populations and were not a result of fecal bacterial contamination, OTUs identified in cattle fecal bacterial communities sampled from the same groups of cattle were removed to create a hidespecific bacterial community (HSO). Bacterial families known to inhabit skin and soil environments dominated the HSO group (Additional file 1: Figure S6), while the original dataset $(\mathrm{AHO})$ was dominated by families found in the digestive tract of mammals (Additional file 1: Figure S5). Alpha diversity metrics indicated an association between low bacterial diversity and EHEC occurrence on cattle hide (Fig. 1, Additional file 1: Figure S7). Because our data is observational, we can only hypothesize about the cause of this low diversity trend with EHEC contamination. For instance, it is difficult to assess whether a genomic adaption within the pathogen enabled it to outcompete autochthonous hide microbiota or whether a disturbance presented EHEC populations with the opportunity to colonize an unoccupied ecological niche within the hide community.

Strains of STEC are pathogens that carry a number of genes that enable them to survive within the low $\mathrm{pH}$ conditions of the stomach and within nutrient-limited environments [44]. Almost $20 \%$ of the E. coli O157 genome is composed of foreign DNA not present in commensal E. coli $\mathrm{K}-12$ genome [45]. This additional reservoir of genomic DNA may provide the pathogen with the ability to survive and compete in novel environments, like cattle hides. However, a healthy commensal microbiome may overcome the genomic plasticity of STEC. Generally, antagonism between bacteria can occur indirectly or directly, via mechanisms such as competitive exclusion, the production of antimicrobial agents, or the modification of the environment to unfavorable conditions [46].

It is thought that in an established microbial community, many if not all of the available environmental niches are filled, generating a protective barrier against colonization by an invading pathogen [11]. Therefore, a diverse bacterial community may prevent EHEC from colonizing in a novel environment. Researchers have found that competitive interaction with enteric bacteria can displace or inhibit the growth of E. coli $\mathrm{O} 157$ in vitro [47-49]. For instance, one study reported the survival of $E$. coli $\mathrm{O} 157$ decreased by 20 - to 30 -fold in the presence of Enterobacter asburiae, a competitor for the carbon and nitrogen substrates used by the pathogen [50]. The reduced diversity that was present in a subset of our hide samples may have enabled the pathogen to gain a foothold in a vacant niche (Fig. 1). Alternatively, commensal hide bacterial species may have inhibited the invasion of EHEC populations more directly through the production of antimicrobial compounds such as organic acids and bacteriocins.

In the AHO dataset, the bacterial OTUs that were at higher relative abundance in the absence of EHEC were Ruminococcus, Brevibacterium, and Nocardioidaceae, while in the HSO dataset, the bacteria at higher relative abundance were Soilbacillus and Streptococcus (Fig. 4). Looking at the types of compounds produced by these bacterial taxa, we can build hypotheses about the 
possible impact these groups may have had on STEC populations. Several Ruminococcus spp. (e.g., Ruminococcus albus, Ruminococcus flavefaciens) can produce fermentation products such as succinate acetate, formate, and ethanol in the gut, some of which have been shown to impact the survival of STEC [51]. Ruminococcus, along with Clostridium and Bacteroides, have also been identified as members of the human microbiota that can inhibit stx2 production and, thus, potentially limit STEC (and EHEC) propagation [52].

Interestingly, a species of Soilbacillus, Solibacillus silvestris, has the ability to degrade $N$-acylhomoserine lactones (AHLs) [53]. AHLs are widely used for the cellular communication phenomena known as quorum sensing in Gram-negative bacteria. E. coli do not produce AHLs themselves, but utilize the AHL receptor of the LuxR family, SdiA. SidA receptors recognize AHLs produced from surrounding bacteria to assess the environment and modulate gene expression as necessary [54]. Previous research has demonstrated that AHL perception in E. coli $\mathrm{O} 157$ is utilized for intestinal colonization in cattle and is speculated to be critical in E. coli survival outside the host $[55,56]$. While the role of AHLs in hide colonization is unknown, S. silvestris and other AHLdegrading bacterial populations may be interfering with E. coli $\mathrm{O} 157$ ability to perceive commensal bacteria, thereby decreasing their chances of a successful invasion.

The other group of OTUs identified as having higher prevalence in EHEC negative samples and lower prevalence in $\mathrm{O} 157$ positive samples were OTUs belonging to the genus Streptococcus. In fact, within the hide-specific dataset, (HSO) seven different Streptococcus OTUs were recognized as having lower relative abundance in samples containing EHEC serogroups (Fig. 4). Certain species of Streptococcus (e.g., Streptococcus thermophilus, Streptococcus bovi, etc.) produce lactic acid. In addition to lowering the $\mathrm{pH}$, lactic acid has been shown to cause permeabilization of the outer membrane of Gramnegative bacteria, including E. coli O157 [57]. Lactic acid-producing bacteria (LAB) have been widely studied as probiotics to inhibit $E$. coli $\mathrm{O} 157$, with the majority of these experiments involving Lactobacillus spp. [58, 59]. High levels of lactic acid bacteria were shown to inhibit the growth of E. coli $\mathrm{O} 157$ in ground beef [60] and experimentally infected cattle saw a decrease in E. coli O157 shedding when given a probiotic culture of Streptococcus bovis and Lactobacillus gallinarum [61]. Additionally, sheep fed a cocktail of probiotic bacteria including several Lactobacillus spp., Streptococcus thermophilus and Enterococcus faecium showed a reduction in non-O157 STEC fecal shedding [62]. Thus, Streptococcus may be a useful bacterium in the prevention of STEC colonization in the gut and on the hide.
Despite being a minor community member on the hide and in feces, EHEC populations appear to correspond with drastically different community compositions. This can result in differences between communities containing different EHEC serogroup populations (e.g., O157 versus non-O157) (Figs. 2 and 3). These data suggest that it may be advisable to incorporate bacterial community profiling data into quantitative models of EHEC risks. Going a step further, future investigations should examine the possible use of individual bacterial strains or mixtures of strains as biotherapeutic agents to mitigate EHEC contamination. Through a more complete mechanistic understanding of how commensal microbes on cattle hides prevent EHEC colonization, we may be able to lessen the food safety risks in beef processing associated with these dangerous human pathogens.

\section{Additional file}

Additional file 1: Supplementary information. (PDF 6379 kb)

Competing interests

The authors declare that they have no competing interests.

\section{Authors' contributions}

JC wrote and edited the manuscript and along with ZD performed all $16 \mathrm{~S}$ rRNA gene sample processing. RMM performed the sequence processing and the majority of bioinformatic analysis. NC performed the sample collection and along with $\mathrm{GL}$ and $\mathrm{ZS}$ was responsible for collecting the serotyping data. RAM, DR, NC, and KEW read, edited, and approved the final manuscript.

\section{Acknowledgements}

This project was supported by the Agriculture and Food Research Initiative Competitive grant no. 2012-68003-30155 from the USDA National Institute of Food and Agriculture.

\section{Author details}

${ }^{1}$ Delaware Biotechnology Institute, University of Delaware, Delaware Biotechnology Inst., 15 Innovation Way, Newark, DE 19711, USA. ${ }^{2}$ College of Veterinary Medicine, Kansas State University, Manhattan, KS, USA. ${ }^{3}$ School of Veterinary Medicine \& Biomedical Sciences, University of Nebraska-Lincoln, Lincoln, NE, USA.

Received: 15 November 2015 Accepted: 10 February 2016

Published online: 22 March 2016

\section{References}

1. Caprioli A, Morabito S, Brugère $H$, Oswald E. Enterohaemorrhagic Escherichia coli: emerging issues on virulence and modes of transmission. Vet Res. 2005; 36:289-311.

2. Hancock D, Besser T, Kinsel M, Tarr P, Rice D, Paros M. The prevalence of Escherichia coli O157:H7 in dairy and beef cattle in Washington State. Epidemiol Infect. 1994;113:199-207.

3. Scallan E, Hoekstra RM, Angulo FJ, Tauxe RV, Widdowson M-A, Roy SL, et al. Foodborne illness acquired in the United States-major pathogens. Emerg Infect Dis. 2011;17(1):7-15.

4. Painter JA, Hoekstra RM, Ayers T, Tauxe RV, Braden CR, Angulo FJ, et al. Attribution of foodborne illnesses, hospitalizations, and deaths to food commodities by using outbreak data, United States, 1998-2008. Emerg Infect Dis. 2013;19:407.

5. Boyce TG, Swerdlow DL, Griffin PM. Escherichia coli O157: H7 and the hemolytic-uremic syndrome. New Engl J Med. 1995;333:364-8. 
6. Rangel JM, Sparling PH, Crowe C, Griffin PM, Swerdlow DL. Epidemiology of Escherichia coli O157: H7 outbreaks, United States, 1982-2002. Emerg Infect Dis. 2005;11:603-9.

7. Riley LW, Remis RS, Helgerson SD, McGee HB, Wells JG, Davis BR, et al. Hemorrhagic colitis associated with a rare Escherichia coli serotype. New Engl J Med. 1983;308:681-5.

8. Brooks JT, Sowers EG, Wells JG, Greene KD, Griffin PM, Hoekstra RM, et al. Non-O157 Shiga toxin-producing Escherichia coli infections in the United States, 1983-2002. J Infect Dis. 2005;192:1422-9.

9. Hughes JM, Wilson ME, Johnson KE, Thorpe CM, Sears CL. The emerging clinical importance of non-0157 Shiga toxin-producing Escherichia coli. Clin Infect Dis. 2006;43:1587-95.

10. Buffie CG, Pamer EG. Microbiota-mediated colonization resistance against intestinal pathogens. Nat Rev Immunol. 2013;13:790-801.

11. Hibbing ME, Fuqua C, Parsek MR, Peterson SB. Bacterial competition: surviving and thriving in the microbial jungle. Nat Rev Microbiol. 2010;8:15-25.

12. Zhao L, Tyler P, Starnes J, Bratcher C, Rankins D, McCaskey T, et al. Correlation analysis of Shiga toxin-producing Escherichia coli shedding and faecal bacterial composition in beef cattle. J Appl Microbiol. 2013;115:591-603.

13. Jiang X, Morgan J, Doyle MP. Fate of Escherichia coli O157: H7 in manureamended soil. Appl Environ Microbiol. 2002;68:2605-9.

14. van Elsas JD, Chiurazzi M, Mallon CA, Elhottovā D, Krištưfek V, Salles JF. Microbial diversity determines the invasion of soil by a bacterial pathogen Proc Natl Acad Sci. 2012;109:1159-64.

15. Vivant A-L, Garmyn D, Maron P-A, Nowak V, Piveteau P. Microbial diversity and structure are drivers of the biological barrier effect against Listeria monocytogenes in soil. PLoS One. 2013;8:e76991.

16. Arthur TM, Bosilevac JM, Brichta-Harhay DM, Guerini MN, Kalchayanand N, Shackelford SD, et al. Transportation and lairage environment effects on prevalence, numbers, and diversity of Escherichia coli O157: $\mathrm{H} 7$ on hides and carcasses of beef cattle at processing. J Food Protect. 2007;70:280-6.

17. Bell R. Distribution and sources of microbial contamination on beef carcasses. J Appl Microbiol. 1997;82:292-300.

18. Collis V, Reid C-A, Hutchison M, Davies M, Wheeler K, Small A, et al. Spread of marker bacteria from the hides of cattle in a simulated livestock market and at an abattoir. J Food Protect. 2004;67:2397-402.

19. Elder RO, Keen JE, Siragusa GR, Barkocy-Gallagher GA, Koohmaraie M, Laegreid WW. Correlation of enterohemorrhagic Escherichia coli 0157 prevalence in feces, hides, and carcasses of beef cattle during processing. Proc Natl Acad Sci. 2000;97:2999-3003.

20. Callaway TR, Carr M, Edrington T, Anderson RC, Nisbet DJ. Diet, Escherichia coli O157: H7, and cattle: a review after 10 years. Curr Issues Mol Biol. 2009;11:67.

21. Keen JE, Elder RO. Isolation of shiga-toxigenic Escherichia coli $\mathrm{O} 157$ from hide surfaces and the oral cavity of finished beef feedlot cattle. J Am Vet Med Assoc. 2002;220:756-63.

22. Loretz M, Stephan R, Zweifel C. Antibacterial activity of decontamination treatments for cattle hides and beef carcasses. Food Control. 2011;22:347-59.

23. Croxen MA, Law RJ, Scholz R, Keeney KM, Wlodarska M, Finlay BB. Recent advances in understanding enteric pathogenic Escherichia coli. Clin Microbiol Rev. 2013;26:822-80.

24. Gould LH, Mody RK, Ong KL, Clogher P, Cronquist AB, Garman KN, et al. Increased recognition of non-O157 Shiga toxin-producing Escherichia coli infections in the United States during 2000-2010: epidemiologic features and comparison with E. coli 0157 infections. Foodborne Pathog Dis. 2013; 10:453-60.

25. U.S. Department of Agriculture FSalS. 2014. Pre-harvest management controls and intervention options for reducing Shiga toxin-producing Escherichia coli shedding in cattle: an overview of current research. http:// www.fsis.usda.gov/wps/wcm/connect/d5314cc7-1ef7-4586-bca2f2ed86d9532f/Reducing-Ecoli-Shedding-in-Cattle.pdf?MOD=AJPERES

26. Dewsbury DM, Renter DG, Shridhar PB, Noll LW, Shi X, Nagaraja T, et al. Summer and winter prevalence of Shiga toxin-producing Escherichia coli (STEC) O26, O45, O103, O111, O121, O145, and O157 in feces of feedlot cattle. Foodborne Pathog Dis. 2015;8:726-32.

27. Stromberg ZR, Baumann NW, Lewis GL, Sevart NJ, Cernicchiaro N, Renter DG, et al. Prevalence of enterohemorrhagic Escherichia coli O26, O45, O103, 0111, 0121, 0145, and 0157 on hides and preintervention carcass surfaces of feedlot cattle at harvest. Foodborne Pathog Dis. 2015;12:631-8.

28. Fadrosh DW, Ma B, Gajer P, Sengamalay N, Ott S, Brotman RM, et al. An improved dual-indexing approach for multiplexed 16S rRNA gene sequencing on the Illumina MiSeq platform. Microbiome. 2014;2:1-7.
29. Caporaso JG, Kuczynski J, Stombaugh J, Bittinger K, Bushman FD, Costello EK, et al. QIIME allows analysis of high-throughput community sequencing data. Nat Methods. 2010;7:335-6.

30. DeSantis TZ, Hugenholtz P, Larsen N, Rojas M, Brodie EL, Keller K, et al. Greengenes, a chimera-checked 16S rRNA gene database and workbench compatible with ARB. Appl Environ Microbiol. 2006;72:5069-72.

31. Edgar RC. Search and clustering orders of magnitude faster than BLAST. Bioinformatics. 2010;26:2460-1.

32. Chao A. Nonparametric estimation of the number of classes in a population Scand J Stat. 1984;1:265-70.

33. Faith DP. Conservation evaluation and phylogenetic diversity. Biolo Conserv 1992;61:1-10.

34. Vázquez-Baeza Y, Pirrung M, Gonzalez A, Knight R. EMPeror: a tool for visualizing high-throughput microbial community data. Structure. 2013;585:20.

35. Lozupone C, Knight R. UniFrac: a new phylogenetic method for comparing microbial communities. Appl Environ Microbiol. 2005;71:8228-35.

36. Shannon P, Markiel A, Ozier O, Baliga NS, Wang JT, Ramage D, et al. Cytoscape: a software environment for integrated models of biomolecular interaction networks. Genome Res. 2003;13:2498-504.

37. Chiller K, Selkin BA, Murakawa GJ. Skin microflora and bacterial infections of the skin. J Investig Dermatol Symp Proc. 2001;6:170-4.

38. Cogen A, Nizet V, Gallo R. Skin microbiota: a source of disease or defence? Brit J Dermatol. 2008:158:442-55.

39. Bibel DJ, Aly R, Bayles C, Strauss WG, Shinefield HR, Maibach HI. Competitive adherence as a mechanism of bacterial interference. Can J Microbiol. 1983;29:700-3.

40. Cogen AL, Yamasaki K, Muto J, Sanchez KM, Crotty Alexander L, Tanios J, et al. Staphylococcus epidermidis antimicrobial delta-toxin (phenol-soluble modulingamma) cooperates with host antimicrobial peptides to kill group A Streptococcus. PLoS One. 2010;5:e8557-7.

41. Cogen AL, Yamasaki K, Sanchez KM, Dorschner RA, Lai Y, MacLeod DT, et al. Selective antimicrobial action is provided by phenol-soluble modulins derived from Staphylococcus epidermidis, a normal resident of the skin. J Invest Dermatol. 2010;130:192-200.

42. Grice EA, Segre JA. The skin microbiome. Nat Rev Microbiol. 2011;9:244-53.

43. Gontcharova V, Youn E, Sun Y, Wolcott RD, Dowd SE. A comparison of bacterial composition in diabetic ulcers and contralateral intact skin. Open Microbiol J. 2010;4:8

44. van Elsas JD, Semenov AV, Costa R, Trevors JT. Survival of Escherichia coli in the environment: fundamental and public health aspects. ISME J. 2011;5:173-83.

45. Hayashi T, Makino K, Ohnishi M, Kurokawa K, Ishii K, Yokoyama K, et al. Complete genome sequence of enterohemorrhagic Eschelichia coli O157: H7 and genomic comparison with a laboratory strain K-12. DNA Res. 2001;8:11-22.

46. Blankenship L. Colonization control of human bacterial enteropathogens in poultry. San Diego, CA; Academic Press: 1991.

47. Stavric S, Buchanan B, Gleeson T. Competitive exclusion of Escherichia coli O157: H7 from chicks with anaerobic cultures of faecal microflora. Lett Appl Microbiol. 1992;14:191-3.

48. Zhao T, Doyle MP, Harmon BG, Brown CA, Mueller PE, Parks AH. Reduction of carriage of enterohemorrhagic Escherichia coli $\mathrm{O} 157$ : $\mathrm{H7}$ in cattle by inoculation with probiotic bacteria. J Clin Microbiol. 1998;36:641-7.

49. Zhao T, Tkalcic S, Doyle MP, Harmon BG, Brown CA, Zhao P. Pathogenicity of enterohemorrhagic Escherichia coli in neonatal calves and evaluation of fecal shedding by treatment with probiotic Escherichia coli. J Food Protect. 2003;66:924-30

50. Cooley MB, Chao D, Mandrell RE. Escherichia coli O157: H7 survival and growth on lettuce is altered by the presence of epiphytic bacteria. J Food Protect. 2006;69:2329-35.

51. Russell JB, Rychlik JL. Factors that alter rumen microbial ecology. Science. 2001:292:1119-22.

52. de Sablet T, Chassard C, Bernalier-Donadille A, Vareille M, Gobert AP, Martin C. Human microbiota-secreted factors inhibit shiga toxin synthesis by enterohemorrhagic Escherichia coli O157: H7. Infect Immun. 2009;77:783-90.

53. Morohoshi T, Tominaga Y, Someya N, Ikeda T. Complete genome sequence and characterization of the $\mathrm{N}$-acylhomoserine lactonedegrading gene of the potato leaf-associated Solibacillus silvestris. J Biosci Bioeng. 2012;113:20-5.

54. Sperandio V. SdiA sensing of acyl-homoserine lactones by enterohemorrhagic E. coli (EHEC) serotype O157: H7 in the bovine rumen Gut Microbes. 2010;1:432-5. 
55. Dziva F, van Diemen PM, Stevens MP, Smith AJ, Wallis TS. Identification of Escherichia coli O157: H7 genes influencing colonization of the bovine gastrointestinal tract using signature-tagged mutagenesis. Microbiology. 2004:150:3631-45.

56. Van Houdt R, Aertsen A, Moons P, Vanoirbeek K, Michiels CW. N-acyl-Lhomoserine lactone signal interception by Escherichia coli. FEMS Microbiol Lett. 2006;256:83-9.

57. Alakomi H-L, Skyttä E, Saarela M, Mattila-Sandholm T, Latva-Kala K, Helander I. Lactic acid permeabilizes Gram-negative bacteria by disrupting the outer membrane. Appl Environ Microbiol. 2000;66:2001-5.

58. Brashears M, Jaroni D, Trimble J. Isolation, selection, and characterization of lactic acid bacteria for a competitive exclusion product to reduce shedding of Escherichia coli 0157: H7 in cattle. J Food Protect. 2003;66:355-63.

59. Peterson R, Klopfenstein TJ, Erickson GE, Folmer J, Hinkley S, Moxley RA, et al. Effect of Lactobacillus acidophilus strain NP51 on Escherichia coli O157: H7 fecal shedding and finishing performance in beef feedlot cattle. J Food Protect. 2007:70:287-91.

60. Vold L, Holck A, Wasteson Y, Nissen H. High levels of background flora inhofibits growth of Escherichia coli O157: H7 in ground beef. Int J Food Microbiol. 2000;56:219-25.

61. Ohya T, Akiba M, Ito $\mathrm{H}$. Use of a trial probiotic product in calves experimentally infected with Escherichia coli O157. JARQ. 2001;35:189-94.

62. Rigobelo E, Karapetkov N, Maestá S, Ávila F, McIntosh D. Use of probiotics to reduce faecal shedding of Shiga toxin-producing Escherichia coli in sheep. Benef Microbes. 2014;6:53-60.

\section{Submit your next manuscript to BioMed Central and we will help you at every step:}

- We accept pre-submission inquiries

- Our selector tool helps you to find the most relevant journal

- We provide round the clock customer support

- Convenient online submission

- Thorough peer review

- Inclusion in PubMed and all major indexing services

- Maximum visibility for your research

Submit your manuscript at www.biomedcentral.com/submit 